\title{
Eccentric/concentric ratios at selected velocities for the invertor and evertor muscles of the chronically unstable ankle
}

\author{
H D Hartsell, S J Spaulding
}

\begin{abstract}
Objective-The use of muscle balancing by the clinician to determine return to activity or discharge of a patient is not a well understood measure. Because of the lack of information on the poorly understood concept of eccentric/concentric (E/C) ratios at the ankle, the purpose was to determine the $\mathrm{E} / \mathrm{C}$ ratios for the invertor and evertor muscles at various velocities in healthy and chronically unstable ankles.

Methods-Ten subjects with healthy ankles and 14 with chronically unstable ankles performed five maximal effort reciprocal eccentric/contraction contractions on an isokinetic dynamometer at four velocities $(60,120,180$, and $240 \%$ s) and for each physiological movement of inversion and eversion. Data were analysed using a two way mixed model analysis of variance with repeated measures, with Tukey's test used for post hoc analysis. Results-Although the chronically unstable ankle was significantly weaker $(p<0.05)$ eccentrically and concentrically for inversion and eversion, the main effect of the $\mathrm{E} / \mathrm{C}$ ratios for the ankle was not significant for either joint motion. The main effect of velocity was significant $(p<0.05)$ for each joint motion, but no significant interaction effects were observed. As velocity increased, the $\mathrm{E} / \mathrm{C}$ ratio increased, except at 180 and $240 \%$ s for either ankle group.

Conclusions-Chronic ankle instability and muscle weakness co-exist. Adequate $E / C$ ratios in the chronically unstable ankle may exist in the absence of normal strength values.

(Br f Sports Med 1999;33:255-258)
\end{abstract}

Physical Therapy Graduate Program, University of Iowa, Iowa City, Iowa 52242, USA

H D Hartsell

University of Western Ontario, London, Ontario, Canada S J Spaulding

Correspondence to: Dr H D Hartsell, Physical Therapy Graduate Program, 2600 Steindler Building, University of Iowa, Iowa City, Iowa 52242, USA.

Accepted for publication 31 March 1999
Current knowledge on the range of this ratio is mostly for the low medium sector of the velocity spectrum because the use of high velocities for the study of eccentric muscle performance is not without risk. Dvir ${ }^{3}$ proposed that, with respect to single joint testing, particularly the knee and shoulder, the E/C ratios derived from low medium test velocities are typically within the range 0.95-2.05. Various authors ${ }^{12568}$ have shown consistent findings for the $\mathrm{E} / \mathrm{C}$ ratios in healthy individuals and, with only a few exceptions, the upper limit has been found to be around 2.0.

Deviations from this range have been reported in pathological conditions. Bennett and Stauber ${ }^{9}$ tested patients with knee problems who showed a deficiency in slow velocity eccentric exercise. They found a particularly low $\mathrm{E} / \mathrm{C}$ ratio of less than 0.85 , and proposed that this was a potential source in patellofemoral problems. The problem was proposed to be related to an error in the neuromotor control of the quadriceps although another more feasible explanation may be selective inhibition of eccentric performance of the quadriceps as the result of pain. This conclusion was debated by Trudelle-Jackson et $a l^{10}$ in 1989. They challenged the lower end of the proposed range claiming that about $50 \%$ of their asymptomatic subjects who were tested eccentrically and concentrically at a slow velocity for the knee had an $\mathrm{E} / \mathrm{C}$ ratio of less than 0.85 . Others have also shown this proposition to be consistently valid even in the presence of pathology. ${ }^{11}$

Interpretation of isokinetic test findings for ankle dysfunction has often been based on the principles of bilateral comparisons or comparison of dysfunctional with control groups. The use of E/C ratios for strength, work, or power is notably absent. ${ }^{37}$ Only one study ${ }^{12}$ was found that investigated the $\mathrm{E} / \mathrm{C}$ ratios for the dorsiflexors of the ankle. Reinking ${ }^{12}$ reported the $\mathrm{E} / \mathrm{C}$ ratios to be 1.45 and 1.50 for velocities of $30 \%$ and $90 \%$ s respectively. These values are somewhat higher than previously quoted for other muscle groups tested under similar conditions. However, Reinking proposed that the higher eccentric strength may reflect the role of dorsiflexor contraction in mediating the loading response during the stance phase of locomotion.

The lack of information on $\mathrm{E} / \mathrm{C}$ ratios at the ankle prompted this investigation of the $\mathrm{E} / \mathrm{C}$ ratios for the invertor and evertor muscles of the ankle at various velocities in healthy and chronically unstable ankles. The hypothesis was that the $\mathrm{E} / \mathrm{C}$ ratios would be significantly different for chronically unstable ankles. 
Table 1 Subject demographics

\begin{tabular}{llll}
\hline & Mean & $S D$ & Range \\
\hline Age (years) & & & \\
$\quad$ Chronic & 26.6 & 4.29 & $23-33$ \\
$\begin{array}{l}\text { Healthy } \\
\text { Height (cm) }\end{array}$ & 26.0 & 3.14 & $23-32$ \\
$\quad$ Chronic & 170.4 & 10.14 & $157.5-185.4$ \\
$\quad$ Healthy & 171.4 & 9.20 & $157.5-185.4$ \\
Weight (kg) & & & \\
$\quad$ Chronic & 67.4 & 7.85 & $54.5-79.5$ \\
Healthy & 73.3 & 14.08 & $55.5-93.2$ \\
\hline
\end{tabular}

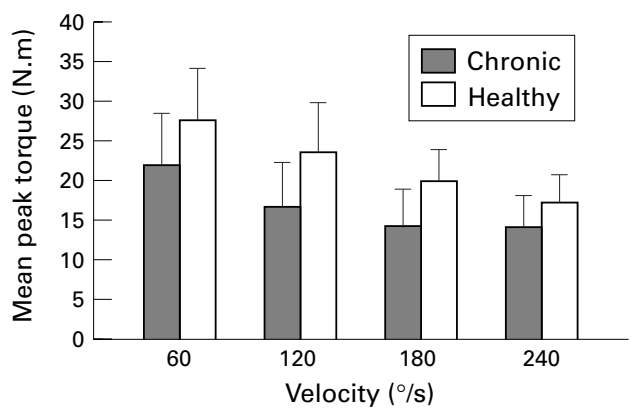

Figure 1 Concentric peak torques for inversion by group.

\section{Experimental design}

A between ankle group (healthy $\times$ chronically unstable) repeated measures design was used. The repeated measures included joint motion (inversion, eversion), muscle contraction type (concentric, eccentric), and velocity $(60,120$, 180 , and $240 \%$ s). The $\mathrm{E} / \mathrm{C}$ ratios used in the analyses were determined from the dependent variable of mean peak torques (N.m). All tests were performed on each subject.

\section{Methods}

SUBJECTS

Table 1 gives the demographics of the subjects. The control group consisted of 10 subjects (five men, five women; mean (SD) age 26.0 (3.14) years) with no prior history of pathology to either lower extremity. The chronic instability group consisted of 14 subjects (seven men, seven women; mean (SD) age 26.6 (4.29) years) who had sustained at least two moderate sprains to the same ankle which required medical intervention and who complained of repeated episodes of "giving way". ${ }^{13}$ No subjects in the chronic instability group had suffered injury to the unstable ankle for at least six months before testing, were undergoing rehabilitation of the ankle, or had any complaints of pain, swelling, or functional limitations. Active range of motion (dorsiflexion/ plantarflexion; inversion/eversion) as measured using a standard goniometer were observed to be within normal limits $\left( \pm 2^{\circ}\right)$ for both groups. No subject was involved in physical activity that exceeded three sessions a week for more than half an hour per session. Written consent was obtained from each subject before testing, and all subjects were screened to ensure that there were no lower extremity neuromuscular or musculoskeletal problems or contraindications for isokinetic testing.

PROTOCOL

Subjects performed a five minute warm up of general range of motion and stretching exer-

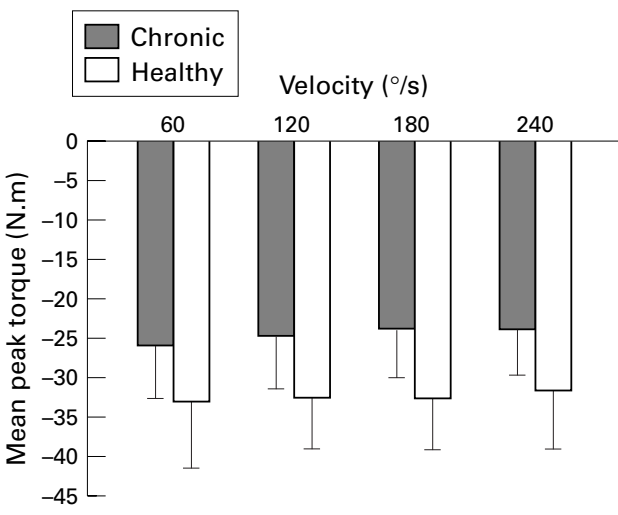

Figure 2 Eccentric peak torques for inversion by group.

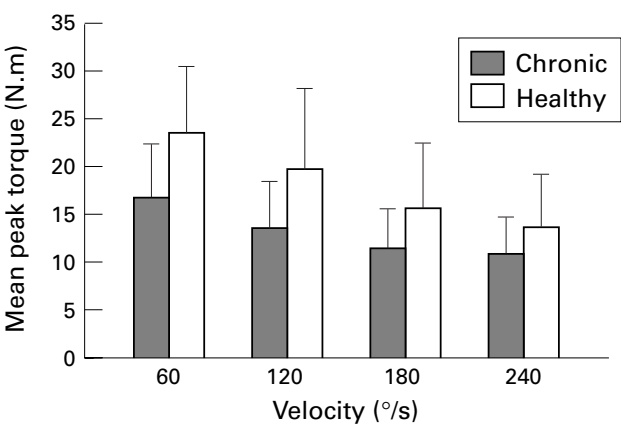

Figure 3 Concentric peak torques for eversion by group.

cises for the joint movements of inversion/ eversion and dorsiflexion/plantarflexion.

After the warm up, they were appropriately positioned on the isokinetic dynamometer (Henley Health Care Systems, Cybex Medical Division, Ronkonkoma, New York, USA) which was calibrated before testing each subject. The ankle joint was positioned in neutral of inversion, and eversion was identified using palpation during passive movements of the talus. ${ }^{14}$ The knee of the test leg was positioned in $80-110^{\circ}$ flexion and the lower leg was parallel to the floor. The ankle joint was positioned in $10-15^{\circ}$ plantarflexion as a consequence of the low cut lace up shoe worn by each subject that simulated a position for inversion injury. ${ }^{15}$ Two straps crisscrossing the dorsum of the foot held it against the footplate. The thigh stabiliser pad and strap secured the distal aspect of the thigh for the test leg and a seatbelt placed around the abdomen secured the torso.

Four submaximal ( $50 \%$ effort) trials were followed by five maximal effort concentric/ eccentric reciprocal contractions at each velocity $(60,120,180$, and $240 \%$ s) and for each physiological movement (inversion, eversion). To ensure that maximal effort was being attained, a cursory review of the five maximal effort torques was performed to verify that all efforts were within $0.5 \mathrm{~N} . \mathrm{m}$ of the peak torque. A two minute rest was permitted between the test for inversion and eversion joint motions to prevent fatigue build up. ${ }^{37}$

\section{DATA ANALYSIS}

Two way (ankle group $\times$ velocity) mixed model analyses of variance with repeated measures of the $\mathrm{E} / \mathrm{C}$ ratios were used separately for joint 


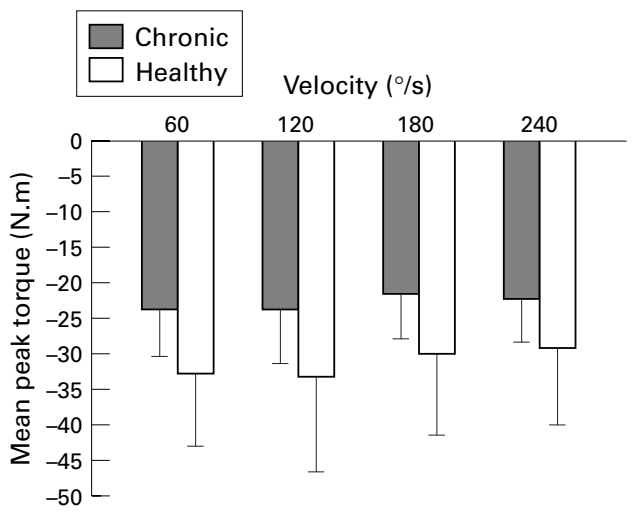

Figure 4 Eccentric peak torques for eversion by group.

motions. Tukey's tests were used for post hoc analysis in the event of significant interaction effects. A significant probability level of 0.05 was used throughout the analyses.

\section{Results}

The chronically unstable ankle was significantly weaker than the healthy ankle, concentrically and eccentrically for both joint motions of inversion ( $p=0.017$ and 0.008 respectively) (figs 1 and 2) and eversion ( $p=0.039$ and 0.024 respectively) (figs 3 and 4). However, the main effect of the $\mathrm{E} / \mathrm{C}$ ratios (table 2) for the ankle was not significant for either joint motion of inversion $(\mathrm{p}=0.713)$ or eversion $(\mathrm{p}=$ 0.746).

A similar pattern was observed for the $\mathrm{E} / \mathrm{C}$ ratios for joint motions of inversion and eversion. The main effect of velocity was significant $(p=0.001$ for each joint motion) but no significant interaction effects were observed. On follow up analysis, as velocity increased, the $\mathrm{E} / \mathrm{C}$ ratio was observed to increase significantly except for the faster velocities of 180 and $240 \%$ s $(p<0.05$ for eversion, $\mathrm{p}<0.002$ for inversion). This was observed for the chronically unstable and healthy ankle groups.

\section{Discussion}

Traditionally, clinicians have used comparisons of the strength values between similar muscle groups on opposite extremities or considered the muscle antagonistic ratios to determine if a patient is rehabilitated. Although technological advances have led to the use of isokinetics to improve evaluation, isokinetic assessment has often been limited to concentric muscle contraction types. ${ }^{16}$ However, we can now expand these measures to include eccentric contractions and focus on the contraction

Table 2 Mean (SD) inversion and eversion $E / C$ ratios and for ankle group $\times$ velocity $\left(60,120,180\right.$, and $\left.240^{\circ} / \mathrm{s}\right)$

\begin{tabular}{|c|c|c|c|c|}
\hline & \multicolumn{4}{|l|}{ Velocity $(\mathrm{C} / \mathrm{s})$} \\
\hline & 60 & 120 & 180 & 240 \\
\hline \multicolumn{5}{|c|}{ Group inversion } \\
\hline Chronic & $1.21(0.23)$ & $1.53(0.27)$ & $1.73(0.35)$ & $1.72(0.33)$ \\
\hline Healthy & $1.19(0.09)$ & $1.40(0.12)$ & $1.64(0.11)$ & $1.83(0.18)$ \\
\hline \multicolumn{5}{|l|}{ Eversion } \\
\hline Chronic & $1.45(0.24)$ & $1.83(0.57)$ & $2.00(0.62)$ & $2.20(0.74)$ \\
\hline Healthy & $1.40(0.21)$ & $1.72(0.29)$ & $1.96(0.23)$ & $2.19(0.36)$ \\
\hline
\end{tabular}

mode dependent order of muscle strength which gives rise to the $\mathrm{E} / \mathrm{C}$ ratio. It has been substantiated that abnormalities in the $\mathrm{E} / \mathrm{C}$ ratio may imply pathology or predispose to injury. $^{31011}$

Muscle weakness and chronic instability at the ankle were found to co-exist. The chronically unstable ankle was significantly weaker than the healthy ankle regardless of muscle contraction type or joint motion. While this observed relation between peroneal muscle weakness and chronic instability supported earlier findings, ${ }^{17}{ }^{18}$ a similar finding was also observed for the invertor muscles which is a new finding. The chronically unstable ankle would appear to be at risk of reinjury.

Subject demographics have often been considered to be a factor in the production of strength output. In this study, the variables of age and height were similar between the groups with healthy and chronically unstable ankles. While there was some variability in weight between the two groups, the differences are not considered to be an issue in the results observed. According to Perrin, ${ }^{7}$ demographic variables are thought to be a factor in the production of strength in the larger joints of the body. However, the ankle is considered to be a small joint and when tested in a gravity-free position is not affected as such.

The eccentric peak torques for the evertor muscles produced for either ankle group were observed to decrease at the higher velocities of 180 and $240 \%$ s, contrary to the established force-velocity relation. ${ }^{19}$ Maximal eccentric contractions may have been diminished by the consecutive eccentric/concentric testing because eccentric contractions are more fatiguing than concentric contractions and the differences are more pronounced at velocities over $150 \%{ }^{7}$ Although the decrements were not statistically significant, clinically they suggest an inability of the evertor muscles to work eccentrically at the faster velocities, which are considered to be more functionally representative of activities of daily living. Ligamentous injury typically occurs when the peroneal muscles are called upon to work eccentrically in response to high velocity movements. ${ }^{20}$ However, at the higher velocity of $240 \%$ shich is functionally relevant, the ability of the evertor muscles to work eccentrically was reduced, causing functional muscle activity around the ankle to be impaired under eccentric and high velocity conditions.

This study supports the idea of a contraction mode dependent order of muscle strength and the magnitude of the moments generated in both contraction modes being velocity dependent. The $\mathrm{E} / \mathrm{C}$ ratios for the joint motions were found to be similar in the two ankle groups and to increase proportionately with increasing velocities, which supports previous research on healthy subjects tested at the elbow ${ }^{513}$ and knee. ${ }^{16}$ As a consequence, our hypothesis was rejected.

Opinions on acceptable $\mathrm{E} / \mathrm{C}$ ratios are diverse. In a summary by Dvir, ${ }^{3}$ it was reported that $\mathrm{E} / \mathrm{C}$ ratios (derived from low to medium test velocities for healthy subjects) using single 
Table 3 Range of $E / C$ ratios in the literature

\begin{tabular}{|c|c|c|c|}
\hline Reference & Foint & Range & Velocity $(\% / s)$ \\
\hline Kramer and MacDermid ${ }^{6}$ & Knee & $1.1-1.5$ & $45-180$ \\
\hline Rizzardo et $a l^{8}$ & Knee & $1.3-1.7$ & $60-180$ \\
\hline Colliander and Tesch ${ }^{5}$ & Knee & $1.2-1.6$ & $30-150$ \\
\hline Griffin $^{1}$ & Elbow & $1.1-1.3$ & $30-120$ \\
\hline Hortobagyi and Katch ${ }^{2}$ & Elbow & $1.4-1.7$ & $30-120$ \\
\hline \multirow[t]{2}{*}{$\mathrm{Dvir}^{3}$} & Shoulder (IR) & $1.1-1.2$ & $60-180$ \\
\hline & Shoulder (ER) & $1.2-1.7$ & $60-180$ \\
\hline \multirow[t]{2}{*}{ Hartsell and Spaulding ${ }^{21}$} & Ankle (Inv) & $1.05-2.00$ & $60-240$ \\
\hline & Ankle (Ever) & $1.17-2.83$ & $60-240$ \\
\hline
\end{tabular}

Inv, inversion; Ever, eversion; IR, internal rotation; ER, external rotation.

joint testing such as the knee and shoulder should be within a range of $0.95-2.05$. Table 3 provides an $\mathrm{E} / \mathrm{C}$ ratio summary along with the results for the healthy subjects in the present study. If the velocity of $240 \% \mathrm{~s}$ is excluded, the $\mathrm{E} / \mathrm{C}$ ratios for the healthy subjects are within acceptable limits. ${ }^{12}$

For the chronically unstable ankle, the mean $\mathrm{E} / \mathrm{C}$ ratios for eversion and inversion joint motions were 1.55 (range $0.80-2.20$ ) and 1.87 (range 1.09-3.67) respectively. The variability observed for the chronically unstable ankle is greater than for the healthy ankle, suggesting inappropriate muscle strength, particularly for the evertor muscles. As the $\mathrm{E} / \mathrm{C}$ ratio increases, either the eccentric force has increased compared with the concentric force or the concentric torque has decreased compared with the eccentric torque. If one reconsiders the forcevelocity relation, the ability of the evertor muscles in the chronically unstable ankle to maintain concentric torque production as velocity increased was inadequate compared with the invertors. These findings may help to explain the high incidence of chronic instability and the susceptibility of the healthy ankle to injury. ${ }^{1521}$ Rehabilitation should include not only concentric muscle strengthening but eccentric muscle strengthening, particularly for the evertors.

Previously, E/C ratios indicative of pathology have been identified for the knee joint only. Bennett and Stauber ${ }^{9}$ studied E/C torque deficits in healthy subjects and patients with anterior knee pain using a test velocity of $30 \%$ and were able to identify a pattern of reduced $\mathrm{E} / \mathrm{C}$ ratio deficits of 0.15 which were predictive of anterior knee pain. Trudelle-Jackson et $a l^{10}$ attempted to determine the $\mathrm{E} / \mathrm{C}$ torque deficits in leg extension. Using the results of Bennett and Stauber, ${ }^{9}$ they set an E/C ratio of 0.85 or less as being predictive of anterior knee pain pathology in healthy subjects, but were unable to identify a pattern suggestive of instability. Although we tested healthy and chronically unstable ankles over a velocity continuum, we also were not able to identify an $\mathrm{E} / \mathrm{C}$ ratio pattern suggestive of instability.

Velocities of up to $160 \%$ for concentric inversion and eversion joint motion testing of the ankle have been reported in the literature..$^{1522}{ }^{23}$ No studies have been reported using eccentric muscles contraction types. This is the first study to have used $240 \%$ s during eccentric and concentric contraction types. Although joint movements during the activities of daily living may exceed this velocity, the information gained on peak torques and $\mathrm{E} / \mathrm{C}$ ratios should still be valuable to the clinician in determining deficits.

\section{CLINICAL RELEVANCE}

Chronic instability and muscle weakness coexist at the ankle and are particularly evident at the higher more functionally relevant velocities. The E/C ratios for the chronically unstable ankle are not significantly different from those for the healthy ankle. Although appropriate balancing of muscle contraction types, as determined by the $\mathrm{E} / \mathrm{C}$ ratios, may exist in the chronically unstable ankle, the clinician should be aware of this existence in the absence of normal strength values when considering a return to activity for the patient.

1 Griffin JW. Differences in elbow flexion torque measured concentrically, eccentrically and isometrically. Phys Ther 1987;67:1205-8.

2 Hortobagyi T, Katch FI. Eccentric and concentric torquevelocity relationships during arm flexion and extension. Eur velocity relationships during arm

F Appl Physiol 1995;60:395-401.
3 Dvir Z. Isokinetics: muscle testing, interpretation and clinical Dvir Z. Isokinetics: muscle testing, interpretation and clin

4 Opplications. New York: Churchill Livingstone, 1995. Olson VL, Smidt GL, Johnston R. The maximum torque
generated by the concentric, isometric and eccentric contractions of the hip abductor muscles. Phys Ther 1972; 52:149-52.

5 Colliander EB, Tesch PA. Bilateral eccentric and concentric torque of quadriceps and hamstring muscles in females and males. Eur f Appl Physiol 1989;59:227-32.

6 Kramer JF, MacDermid J. Isokinetic measures during concentric-eccentric cycles of the knee extensors. Australian fournal of Physiotherapy 1989;35:9-14.

7 Perrin DH. Isokinetic exercise and assessment. Champaign, IL: Human Kinetics Publishers, 1993.

8 Rizzardo M, Wessel J, Bay G. Eccentric and concentric torque and power of the knee extensors of females. Can $\mathcal{F}$ Sport Sci 1988;3:166-9.

9 Bennett JG, Stauber WT. Evaluation and treatment of anterior knee pain using eccentric exercise. Med Sci Sports Exerc 1986;18:526-30.

10 Trudelle-Jackson E, Meske N, Highenboten C, et al. Eccentric/concentric torque deficits in the quadriceps muscle. F Orthop Sports Phys Ther 1989;11:142-5.

11 Conway A, Malone T, Conway P. Patellar alignment/ tracking: effect on force output and perceived pain. Isokinetics and Exercise Science 1992;2:9-17.

12 Reinking MF. The effect of concentric and eccentric training on the strengthening of tibialis anterior. Isokinetics and Exercise Science 1991;1:193-210.

13 Freeman $M$. Instability of the foot after injuries to the lateral ligaments of the ankle. F Bone foint Surg [Br] 1965;47:66977 .

14 Donatelli R. The biomechanics of the foot and ankle. 2nd ed. Philadelphia: FA Davis Co, 1996.

15 Wong DLK, Glasheen-Wray M, Andrews LF. Isokinetic evaluation of the ankle invertors and evertors. F Orthop
Sports Phys Ther 1984;5:246-52.

16 Sapega AA. Muscle performance evaluation in orthopedic Sapega AA. Muscle performance evaluation in orthor
practice. F Bone foint Surg [Am] 1990;72:1562-74.

17 Bosien WR, Staples OS, Russell SW. Residual disability following acute ankle sprain. F Bone foint Surg [Am] 1955;37: 1237-43.

18 Tropp $\mathrm{H}$. Pronator muscle weakness in functional instability of the ankle joint. Int $\mathcal{F}$ Sports Med 1986;7:291-4.

19 Hill AV. The mechanics of active muscle. Proc R Soc Lond B Biol Sci 1953;141:104-17.

20 Lentell G, Katzman L, Walters M. The relationship between muscle function and ankle instability. F Orthop Sports Phys Ther 1990;11:605-11.

21 Hartsell HD, Spaulding SJ. Effectiveness of external orthotic support on passive soft tissue resistance of the chronically unstable ankle. Foot Ankle Int 1997;18:144-50.

2 Cawthorn M, Cummings G, Walker JR, et al. Isokinetic measurement of foot invertor and evertor forces in three positions of plantarflexion and dorsiflexion. F Orthop Sports
Phys Ther 1991;14:75-81.

23 Leslie M, Zachazewski J, Browne P. Reliability of isokinetic torque values for ankle invertors and evertors. F Orthop Sports Phys Ther 1990;12:612-16. 